\title{
Effect of Mineral, Organic and Bio-fertilization on Growth and Production of Moringa (Moringa oleifera, L.) Plants
}

\author{
Radwan, F.I. ${ }^{(1)}$, A.I. A. Abido(1) ${ }^{(1)}$ E.H. Shaben ${ }^{(2)}$ and N. A. Abdel Gabr \\ ${ }^{(1)}$ Plant Production Dept. Faculty of Agriculture (Saba Basha) Alexandria University \\ (2) Medicinal and Aromatic Res. Dept. A.R.C. Alexandria, Egypt of Medicinal and \\ Aromatic plants
}

\begin{abstract}
Two filed experiments were carried out at the Experimental Farm, Faculty of Agriculture (Saba Basha), Alexandria University at Abees region, Alexandria, Egypt during the two growing seasons of 2014 and 2015 to study the effect of mineral, organic and biofertilization on growth and productivity of moringa plants (Moringa oleifera, Lam). The experimental design was split plot were three replicates. The main plot were conducted for the five combination of organic manure plus mineral fertilizer of $100 \%$ organic, $75 \%$ organic manure $+25 \%$ mineral, $50 \%$ organic manure $+50 \%$ mineral, $25 \%$ organic manure $+75 \%$ mineral and $100 \%$ mineral), while, the four bio-fertilization treatments were uninoculation, phosphorein, A- mycorrhizal and cerealine were arranged in the sub-plot. The main results could be summarized as follows: (1) The application of $75 \%$ organic manure $+25 \%$ mineral; gave the highest mean values of all studied characters, (2) the application of $75 \%$ organic manure $+25 \%$ mineral with A- mycorrhizal inoculation was the best combination to obtain the highest mean values of plant height, stem length, stem diameter, number of branches /plant, fresh and dry weights/plant, $\mathrm{K}(\%)$, total carbohydrate (\%) and vitamin (C). However, all traits under study increased significantly due to inoculation treatments over the application $100 \%$ mineral with uninoculation treatments.
\end{abstract}

Keywords: Moringa oleifera, vegetative growth, inorganic, organic and bio-fertilization.

\section{INTRODUCTION}

Moringa (Moringa oleifera) is well known for its multi-purpose attributes, wide actaplability and case of establishment. Every part of the plant is of food value, moringa leaves contain seven times more vitamin- $C$ than oranges, four times more calcium than milk, four times more vitamin-A than carrot, three times more potassium than banana and two times more milk. Hence, it is considered as a powerhouse of nutritional value (Morton, 1991). The seeds are also used for oil productions; this oil is used in art, cosmetics and medicine; and can be consumed as food. Bio-fertilizers are microbial inoculants used for application to either seeds or soil for increasing soil fertility with objective of increasing the number of such micro- organisms and to accelerate ecertain microbial processes (Mazher, et al. 2014).

Fertilization is one of the most important factors limiting the productivity of plants. The intensive use of expensive mineral fertilizers in recent gears results in environmental pollution problems. However, chemical fertilizers at extremely high rates for a long period decreased the potential activity of microflora. (Adeoye et al., 2005).

Additionally, organic manures in the form of compost, animal manure, farmyard manure (FYM) and green manure organic materials are generally added to soils to improve their physical and chemical properties. They enhance 
the soil fertility by their composition of macro and micro-elements, amino acid, organic acids, sugars and organic matter (Abou El-Fadi, 1968). Furthermore, biofertilization is an important factor being used to produce products without some mineral fertilizer that cause environmental pollution problems and high rates of it leads in decrease the potential activity of microflora and the mobility of organic matters. Hence, the attention has been focused on the researches of biofertilization to safe alternative specific chemical fertilizers. Biofertilizers play vital role of increasing the number of microorganisms and accelerate certain of microbial process in the rhizosphere of inoculated soil of plants which can change the available form of some nutrients to be plants (Anjorin et al., 2010; Adebayo et al., 2011; Attia et al., 2014). This research, however, in an attempt to find out the best fertilization treatments, i.e. mineral fertilizer plus organic manure and biofertilizer on the vegetative growth and chemical composition of moringa (Moringa oleifera).

\section{MATERIALS AND METHODS}

The present investigation was carried out during both seasons of 2014 and 2015 at Abees Experimental Farm of the Faculty of Agricultural (Saba Basha), Alexandria University. A filed experimental was designed to study the effect of mineral, organic and bio-fertilization on growth and production of Moringa plants.

Some physical and chemical properties of the experimental field soil and organic matter during the two seasons were done and the data are shown in Tables (1 and 2).

Regarding the cultivated of Moringa oleifera plant look place the research and production station, Cairo (National Research Center). However, planted in $2.5 \times 2.5$ meter space. Mineral fertilizer was applied at $600 \mathrm{~g} /$ tree of ammonium nitrate $(33.5 \% \mathrm{~N}), 250 \mathrm{~g} /$ tree of calcium super-phosphate $\left(15.5 \% \mathrm{P}_{2} \mathrm{O}_{5}\right)$ and $300 \mathrm{~g} /$ tree of potaium sulphate $\left(50 \% \mathrm{~K}_{2} \mathrm{O}\right) .1 .5 \mathrm{~kg}$ sheep manure with $400 \mathrm{~g}$ biofertilizer (phosphorein and cerealine) and and cerealine) liter of Amycorrhizal, and rate of calcium superphosphate were mixed with $0.15 \mathrm{~m}$ depth of top soil around the tree trunk at one dose at March, while nitrogen and potassium fertilizer were applied in three equal doses at April, May and June.

The applied treatments were a follow:

1.Fertilization

$100 \%$ organic

$75 \%$ organic manure $+25 \%$ mineral

$50 \%$ organic manure $+50 \%$ mineral

$25 \%$ organic manure $+75 \%$ mineral

$100 \%$ mineral 
Table (1). Some physical and chemical properties of the experimental soil in 2014 and 2015 seasons

\begin{tabular}{|c|c|c|}
\hline \multirow{2}{*}{ Soil properties } & \multicolumn{2}{|c|}{ Season } \\
\hline & 2014 & 2015 \\
\hline \multicolumn{3}{|l|}{ A) Mechanical analyses : } \\
\hline Clay \% & 42.50 & 43.00 \\
\hline Sand \% & 16.50 & 15.80 \\
\hline Silt $\%$ & 41.00 & 41.20 \\
\hline Soil texture & \multicolumn{2}{|c|}{ Clay loam soil } \\
\hline \multicolumn{3}{|l|}{ B) Chemical properties } \\
\hline $\mathrm{pH}(1: 1)$ & 7.60 & 7.80 \\
\hline $\mathrm{EC}(\mathrm{dS} / \mathrm{m})$ & 2.20 & 2.30 \\
\hline \multicolumn{3}{|c|}{ 1) Soluble cations (1:2) (cmol/kg soil) } \\
\hline $\mathrm{K}^{+}$ & 0.90 & 0.92 \\
\hline $\mathrm{Ca}^{++}$ & 4.20 & 4.25 \\
\hline $\mathrm{Mg}^{++}$ & 3.10 & 3.20 \\
\hline $\mathrm{Na}^{++}$ & 8.20 & 8.15 \\
\hline \multicolumn{3}{|c|}{ 2) Soluble anions (1:2) (cmol/kg soil) } \\
\hline $\mathrm{CO}_{3}^{--}+\mathrm{HCO}_{3}^{-}$ & 2.80 & 2.70 \\
\hline $\mathrm{Cl}^{-}$ & 11.30 & 11.50 \\
\hline $\mathrm{SO}_{4}^{-}$ & 0.48 & 0.50 \\
\hline Calcium carbonate (\%) & 7.80 & 7.90 \\
\hline Total nitrogen (\%) & 0.48 & 0.49 \\
\hline Available phosphate $(\mathrm{mg} / \mathrm{kg})$ & 3.60 & 3.70 \\
\hline Organic matter $(\%)$ & 0.95 & 0.90 \\
\hline
\end{tabular}

Table (2). Analysis of the applied organic manure (sheep manure).

\begin{tabular}{lc}
\hline Properties of organic manure & Value \\
\hline $\mathrm{pH}$ & 7.2 \\
$\mathrm{O} . \mathrm{M} \%$ & 35.5 \\
O.C \% & 22.6 \\
Total N\% & 2.05 \\
Total P\% & 1.20 \\
Total K\% & 1.50 \\
C/N ratio & $13.0: 1$ \\
\hline
\end{tabular}

1.Biofertilizers treatments were randomly distributed in the sub plot a follows:

- Without inoculation (control)

- Inoculation with cerealine: An inoculate for all crops containing of Azospirillum pp. (10 cell/g), Azotobacter chrooococum.

- Inoculation with phosphorein: An inoculate for all crops containing of (Bacillus megatherium) soluble calcium phosphate. These inoculations are produced by the General Organization for Agriculture Equalization Ministry of Agriculture and land Reclamination Egypt (Ismali et al., 2009). 
- Inoculation of A-mycorrhizal fungi: inoculants for Moringa with fungi (Glomus mcrocarpium) strain from plant production Dept. (Saba Basha) Alex. Univ., at a rate of $250 \mathrm{ml}$ of infected roots and was mixed with tress of Moringa plants.

The plants were harvested 3 times per seasons i.e. August $10^{\text {th }}$ September $10^{\text {th }}$ in the first and second seasons by cutting the vegetative parts.

\section{The following data of vegetative growth were recorded:}

Plant height $(\mathrm{cm})$, stem length $(\mathrm{cm})$, stem diameter $(\mathrm{cm})$, number of branches /plant, shoot fresh weight $(\mathrm{g})$ and shoot dry weight $(\mathrm{g})$.

\section{The chemical compositions were recorded as following:}

For these analyses, the leaves were dried at $70^{\circ} \mathrm{C}$ for $48 \mathrm{hr}$., and ground. Leaves $(0.5 \mathrm{~g})$ were digested with sulphuric acid and hydrogen peroxide $\mathrm{H}_{2} \mathrm{SO}_{4}+\mathrm{H}_{2} \mathrm{O}_{2}$ according to the method of (Lowther, 1980) and the following determining were carried out in the digested solution to determine the following:

\section{- Nitrogen content (N\%)}

Nitrogen was determined in digested plant material colorimetrically by Nessler's method (Chapman and Pratt, 1978). Nessler solution (35 g Kl/100 ml d.w. + 20g $\mathrm{HgCl}_{2} / 500 \mathrm{ml} \mathrm{d.w.)}+120 \mathrm{~g} \mathrm{NaOH} / 250 \mathrm{ml}$ d.w. Reading was achieved using wave length of $420 \mathrm{~nm}$ and $\mathrm{N}$ was determined as percentage as follows:

$\% \mathrm{~N}=\mathrm{NH}_{4} \% \times 0.776485$

\section{- Phosphorus content (P \%)}

Phosphorus was determined by the Vanadomolyate yellow method as given by Jackson (1973) and the intensity of color developed was read in spectrophotometer at $405 \mathrm{~nm}$.

\section{- Potassium content ( $\mathrm{K} \%)$}

Potassium was determined according to the method described by method Jackson (1973) using Beckman Flame photometer.

- Total soluble carbohydrates were determined, quantitatively, in the herb of sage by Anthron method according to Yemm and Willis (1954) as follows:

Extraction was carried out by grinding dry matter in Mahadavaine buffer (sodium citrate buffer, $\mathrm{pH}$ 6.8). Extracts were homogenized for 3 minutes and centrifuged at $4000 \mathrm{rpm}$ for $15 \mathrm{~min}$. the supernatant was then used to determine total soluble carbohydrates.

- Protein was determined by estimating the total nitrogen in the herbs and multiplied by 6.25 to obtain the percentage according to AOAC (1990). 
- The ascorbic acid content of the juice was determined by titration with 4, 6 dichloro phenol-indo-phenol (AOAC, 1984) and calculated as milli-grams per $100 \mathrm{ml}$ of juice.

The obtained data were statistically analyzed according to Gomez and Gomez (1984). The least significantly differences test (L.S.D.) at 0.05 was used in compare between means of the different treatments.

\section{RESULTS AND DISCUSSIONS}

\section{A) Vegetative growth}

The obtained results, given in Tables (3, 4 and 5) clearly show that combination of mineral plus organic manure fertilizer exhibited a significant effect on all estimated traits at the achieved three cuts during both seasons. Application of $75 \%$ organic manure $+25 \%$ mineral, significantly, increased plant height, stem length, stem diameter, number of branches /plant, shoot fresh and shoot dry weight/plant at the three cuts during both seasons. These results may be due to the nutritional benefits of organic manure which include improvement of soil fertility, water holding capacity and organic matter and response to organic manure attributed to increasing nitrogen nutrition as indicated by increased concentration in plant tissues (Dania et al., 2014).

Inoculation of A- mycorrhizal fungi, significantly, increased plant height, stem length, stem diameter, number of branches /plant, shoot fresh and shoot dry weight/plant at three cuts during both seasons in comparison to uninoculation treatments (control). It could be concluded that A- mycorrhizal fungi inoculation treatment promoted the production of moringa growth. However, these events could be attributed to more adsorption of nutrients which reflected more on growth, more cell division and enlargement more of tissue and organs and plant elongation. Also, the phosphate solubilizing bacteria and nitrogen fixing may increases. The synthesis of endogenous phytohormones, i.e. IAA, GAs and CKs which play an important role in formation of mass active root system which allow more nutrients uptake. The previous results agree, more or less, with the findings of Rajendrn et al. (2000) on Cassuasin equisetifolia, Manorama et al. (2007) on Acaci mellifera and Attia et al. (2014) on Moringa oleifera.

The interaction between organic manure + mineral and bio-fertilization was significant and affected all traits at the three cuts during both seasons (Tables 3, 4 and 5). Tables (6 and 7) decleard, the application of $75 \%$ organic manure $+25 \%$ mineral, resulted in the highest shoot fresh and shoot dry weight mean values with inoculation with A- mycorrhizal. 
Table (3). Plant height (cm) and stem length $(\mathrm{cm})$ as affected by mineral-organic and biofertilization at the three cuts in 2014 and 2015 seasons.

\begin{tabular}{|c|c|c|c|c|c|c|c|c|c|c|c|c|}
\hline \multirow{3}{*}{ Treatments } & \multicolumn{6}{|c|}{ Plant height (cm) } & \multicolumn{6}{|c|}{ Stem length (cm) } \\
\hline & \multicolumn{3}{|c|}{2014 Season } & \multicolumn{3}{|c|}{2015 Season } & \multicolumn{3}{|c|}{2014} & \multicolumn{3}{|c|}{2015} \\
\hline & $1^{\text {st }}$ cut & $2^{\text {nd }}$ cut & $3^{\text {th }}$ cut & $1^{\text {st }}$ cut & $2^{\text {nd }}$ cut & $3^{\text {th }}$ cut & $1^{\text {st }}$ cut & $2^{\text {nd }}$ cut & $3^{\text {th }}$ cut & $1^{\text {st }}$ cut & $2^{\text {nd }}$ cut & $3^{\text {th }}$ cut \\
\hline \multicolumn{13}{|l|}{ A) Mineral + Organic } \\
\hline $100 \%$ Organic & $100.24 \mathrm{e}$ & $111.65 d$ & $124.10 \mathrm{~cd}$ & $111.65 d$ & $121.05 d$ & $134.83 d$ & $71.70 b$ & $89.30 b$ & $111.47 \mathrm{~b}$ & $79.14 b$ & $98.37 b$ & $123.69 b$ \\
\hline $75 \%$ org. $+25 \%$ mineral & $106.25 a$ & $118.06 a$ & $131.18 a$ & $118.04 a$ & $131.18 a$ & $115.75 a$ & $79.16 \mathrm{a}$ & $98.95 a$ & $123.70 a$ & $87.95 a$ & $109.94 a$ & $137.42 \mathrm{a}$ \\
\hline $50 \%$ org. $+50 \%$ mineral & $102.30 \mathrm{~b}$ & $114.75 b$ & $127.12 b$ & $114.42 b$ & $127.13 b$ & $141.25 b$ & $64.09 c$ & $80.15 c$ & $100.19 c$ & $71.21 \mathrm{c}$ & $89.05 c$ & $111.32 c$ \\
\hline $25 \%$ org. $+75 \%$ mineral & $101.46 c$ & $112.75 c$ & $125.27 \mathrm{c}$ & $112.88 \mathrm{c}$ & $125.38 c$ & $139.16 c$ & $57.67 d$ & $72.09 d$ & $90.17 d$ & $64.13 d$ & $80.08 d$ & $100.09 d$ \\
\hline $100 \%$ mineral & $100.72 d$ & $111.92 \mathrm{~cd}$ & $123.54 d$ & $111.91 d$ & $125.18 c$ & $137.25 c$ & $52.23 e$ & $64.92 \mathrm{e}$ & $81.15 \mathrm{e}$ & $58.03 e$ & $73.13 \mathrm{e}$ & $90.16 \mathrm{e}$ \\
\hline LSD 0.05 & 0.44 & 1.05 & 1.25 & 0.40 & 1.70 & 2.05 & 3.10 & 4.50 & 6.30 & 3.70 & 4.70 & 6.90 \\
\hline \multicolumn{13}{|l|}{ B) Bio-fertilization } \\
\hline Unin & $92.44 d$ & $102.73 d$ & $114.12 d$ & $102.71 d$ & $114.12 d$ & $126.73 d$ & $60.66 d$ & $75.85 d$ & $94.96 d$ & $67.43 d$ & $84.88 \mathrm{c}$ & $105.10 d$ \\
\hline Phosphorein & $101.43 b$ & $116.26 b$ & $125.89 b$ & $116.23 b$ & $129.85 b$ & $143.33 b$ & $66.27 \mathrm{~b}$ & $82.45 b$ & $103.08 b$ & $73.56 b$ & $93.61 \mathrm{~b}$ & $114.53 b$ \\
\hline Mycorrhizal & $112.52 a$ & $125.03 a$ & $138.92 a$ & $124.96 a$ & $138.92 a$ & $154.33 a$ & $69.51 \mathrm{a}$ & $86.71 \mathrm{a}$ & $108.04 a$ & $76.89 a$ & $96.13 a$ & $120.14 a$ \\
\hline Cerealine & $99.92 e$ & $111.29 c$ & $122.70 c$ & $111.15 c$ & $123.36 \mathrm{c}$ & $136.33 c$ & $63.43 c$ & $79.29 c$ & $99.11 \mathrm{c}$ & $70.49 c$ & $84.99 c$ & $110.06 c$ \\
\hline LSD 0.05 & 1.02 & 2.10 & 2.30 & 1.50 & 2.20 & 2.50 & 2.40 & 3.10 & 3.90 & 2.20 & 3.30 & 4.10 \\
\hline \multicolumn{13}{|l|}{ Interaction } \\
\hline AxB & * & * & * & * & * & * & * & * & * & * & * & * \\
\hline
\end{tabular}

Means of each factor designated by the same letter not significantly different at $5 \%$ using least significant difference at $5 \%$ level using (L.S.D.) test

*: Significant at 0.05 level of probability. 
Table (4). Stem diameter (cm) and number of branches/plant as affected by mineral-organic and biofertilization at the three cuts in 2014 and 2015 seasons.

\begin{tabular}{|c|c|c|c|c|c|c|c|c|c|c|c|c|}
\hline \multirow{3}{*}{ Treatments } & \multicolumn{6}{|c|}{ Stem diameter (cm) } & \multicolumn{6}{|c|}{ Number of branches/plant } \\
\hline & \multicolumn{3}{|c|}{2014 Season } & \multicolumn{3}{|c|}{2015 son } & \multicolumn{3}{|c|}{2014} & \multicolumn{3}{|c|}{2015} \\
\hline & $1^{\text {st }}$ cut & $2^{\text {nd }}$ cut & $3^{\text {th }}$ cut & $1^{\text {st }}$ cut & $2^{\text {nd }}$ cut & $3^{\text {th }}$ cut & $1^{\text {st }}$ cut & $2^{\text {nd }}$ cut & $3^{\text {th }}$ cut & $1^{\text {st }}$ cut & $2^{\text {nd }}$ cut & $3^{\text {th }}$ cut \\
\hline \multicolumn{13}{|l|}{ A)Mineral + Organic } \\
\hline $100 \%$ Organic & $1.97 b$ & $2.20 \mathrm{~b}$ & $3.15 b$ & $2.20 \mathrm{~b}$ & $2.44 b$ & $3.52 b$ & $8.59 c$ & $9.70 b$ & $10.77 b$ & $9.68 b$ & $10.76 b$ & $11.96 b$ \\
\hline $75 \%$ org. $+25 \%$ mineral & $2.20 \mathrm{a}$ & $2.45 a$ & $3.50 \mathrm{a}$ & $2.44 a$ & $2.72 a$ & $3.88 a$ & 8.93a & $9.93 a$ & 11.03a & 9.93a & $11.03 a$ & $12.26 a$ \\
\hline $50 \%$ org. $+50 \%$ mineral & 1.99ab & $2.02 \mathrm{c}$ & $2.86 c$ & $2.21 b$ & $2.20 c$ & $3.16 c$ & $8.78 b$ & $9.75 b$ & $10.84 b$ & $9.75 b$ & $10.83 b$ & $12.04 b$ \\
\hline $25 \%$ org. $+75 \%$ mineral & $1.61 d$ & $1.79 d$ & $2.57 d$ & $1.79 d$ & $1.99 d$ & $2.85 d$ & $7.89 d$ & $8.78 d$ & $9.75 c$ & $8.86 c$ & $9.75 c$ & $10.83 c$ \\
\hline $100 \%$ mineral & $1.66 \mathrm{c}$ & $1.84 \mathrm{~d}$ & $2.31 \mathrm{e}$ & $1.86 \mathrm{c}$ & $1.88 \mathrm{~d}$ & $2.57 \mathrm{e}$ & $7.24 \mathrm{e}$ & $7.89 \mathrm{c}$ & $8.69 d$ & $8.04 d$ & $8.77 d$ & $9.74 d$ \\
\hline LSD 0.05 & 0.03 & 0.07 & 0.12 & 0.05 & 0.13 & 0.25 & 0.06 & 0.10 & 0.16 & 0.11 & 0.17 & 0.19 \\
\hline \multicolumn{13}{|l|}{ B) Bio-fertilization } \\
\hline Uninoculation & $1.30 \mathrm{~d}$ & $1.44 d$ & $2.07 d$ & $1.44 d$ & $1.30 \mathrm{~d}$ & $2.29 d$ & $7.11 d$ & $7.90 \mathrm{~d}$ & $8.79 d$ & $7.91 d$ & $8.78 d$ & $9.76 \mathrm{~d}$ \\
\hline Phosphorein & $2.01 b$ & $2.26 b$ & $3.21 b$ & $2.24 b$ & $2.49 b$ & $3.56 b$ & $8.60 b$ & $9.55 b$ & $10.61 b$ & $9.55 b$ & $10.61 b$ & $11.79 b$ \\
\hline Mycorrhizal & $2.36 \mathrm{a}$ & $2.63 a$ & $3.76 a$ & $2.62 a$ & $2.91 \mathrm{a}$ & $4.17 a$ & $9.45 a$ & $10.46 a$ & $11.55 a$ & $10.51 a$ & $11.62 a$ & $12.91 \mathrm{a}$ \\
\hline Cerealine & $1.88 \mathrm{c}$ & $1.90 \mathrm{c}$ & $2.47 \mathrm{c}$ & $2.08 \mathrm{c}$ & $1.97 \mathrm{c}$ & $2.74 \mathrm{c}$ & $8.08 \mathrm{e}$ & $8.90 c$ & $9.89 c$ & $9.05 c$ & $9.89 c$ & $10.99 c$ \\
\hline LSD 0.05 & 0.04 & 0.11 & 0.20 & 0.14 & 0.23 & 0.30 & 0.11 & 0.13 & 0.18 & 0.20 & 0.25 & 0.40 \\
\hline \multicolumn{13}{|l|}{ Interaction } \\
\hline$A \times B$ & * & * & * & * & * & * & * & * & * & * & * & * \\
\hline
\end{tabular}

Means of each factor designated by the same letter not significantly different at $5 \%$ using least significant difference at $5 \%$ level using (L.S.D.) test *: Significant at 0.05 level of probability. 
Table (5). Fresh of shoot weight $(\mathrm{g})$ and shoot dry weight $(\mathrm{g})$ as affected by mineral-organic and biofertilization at the three cuts in 2014 and 2015 seasons.

\begin{tabular}{|c|c|c|c|c|c|c|c|c|c|c|c|c|}
\hline \multirow{3}{*}{ Treatments } & \multicolumn{6}{|c|}{ Shoot fresh weight (g) } & \multicolumn{6}{|c|}{ Shoot dry weight (g) } \\
\hline & \multicolumn{3}{|c|}{2014 Season } & \multicolumn{3}{|c|}{2015 son } & \multicolumn{3}{|c|}{2014} & \multicolumn{3}{|c|}{2015} \\
\hline & $1^{\text {st }}$ cut & $2^{\text {nd }}$ cut & $3^{\text {th }}$ cut & $1^{\text {st }}$ cut & $2^{\text {nd }}$ cut & $3^{\text {th }}$ cut & $1^{\text {st }}$ cut & $2^{\text {nd }}$ cut & $3^{\text {th }}$ cut & $1^{\text {st }}$ cut & $2^{\text {nd }}$ cut & $3^{\text {th }}$ cut \\
\hline \multicolumn{13}{|l|}{ A) Mineral + Organic } \\
\hline $100 \%$ Organic & $81.27 b$ & $101.85 b$ & $127.35 b$ & $90.57 b$ & $113.35 b$ & $141.53 b$ & $16.30 \mathrm{~b}$ & $20.38 b$ & $25.48 b$ & $18.19 b$ & $22.64 b$ & $28.31 b$ \\
\hline $75 \%$ org. $+25 \%$ mineral & $90.58 a$ & $113.22 \mathrm{a}$ & $136.41 \mathrm{a}$ & $100.64 a$ & $125.63 a$ & $154.45 a$ & $18.11 \mathrm{a}$ & $22.64 a$ & $29.06 a$ & $20.14 a$ & $25.16 \mathrm{a}$ & $31.448 \mathrm{a}$ \\
\hline $50 \%$ org. $+50 \%$ mineral & $73.36 c$ & $91.70 c$ & $114.64 c$ & $86.51 c$ & $101.94 c$ & $127.31 \mathrm{c}$ & $14.67 c$ & $18.34 c$ & $22.93 c$ & $16.30 c$ & $19.54 c$ & $25.48 c$ \\
\hline $25 \%$ org. $+75 \%$ mineral & $66.72 d$ & $82.62 d$ & $103.18 d$ & $73.47 d$ & $91.54 d$ & $114.63 d$ & $13.20 d$ & $16.51 \mathrm{~d}$ & $20.72 d$ & $14.67 d$ & $18.34 d$ & $22.93 d$ \\
\hline $100 \%$ mineral & $59.43 e$ & $74.28 \mathrm{e}$ & $93.61 \mathrm{e}$ & 66.02 & $82.70 \mathrm{e}$ & $103.17 e$ & $11.83 \mathrm{e}$ & $14.85 \mathrm{e}$ & $18.59 \mathrm{e}$ & $13.19 \mathrm{e}$ & $16.50 \mathrm{e}$ & $21.48 \mathrm{e}$ \\
\hline LSD 0.05 & 3.10 & 5.10 & 6.20 & 3.60 & 5.50 & 7.20 & 0.85 & 1.10 & 1.18 & 1.03 & 1.15 & 1.30 \\
\hline \multicolumn{13}{|l|}{ B) Bio-fertilization } \\
\hline Uninoculation & $66.04 d$ & $83.62 d$ & $103.20 d$ & $73.38 d$ & $91.70 d$ & $114.66 \mathrm{~d}$ & $13.29 d$ & $16.51 d$ & $20.64 d$ & $14.67 d$ & $17.67 d$ & $22.93 d$ \\
\hline Phosphorein & $75.79 b$ & $94.74 b$ & $118.40 \mathrm{~b}$ & $84.21 b$ & $105.23 b$ & $131.59 b$ & $15.16 b$ & $18.94 b$ & $23.75 b$ & $16.76 b$ & $21.05 b$ & $26.35 b$ \\
\hline Mycorrhizal & $83.81 \mathrm{a}$ & $104.77 a$ & $130.94 a$ & $93.12 a$ & $116.40 \mathrm{a}$ & $145.45 a$ & $16.76 a$ & $20.91 a$ & $26.20 a$ & $18.62 a$ & $23.18 \mathrm{a}$ & $29.78 a$ \\
\hline Cerealine & $71.07 c$ & $88.84 \mathrm{c}$ & $111.05 c$ & $78.97 c$ & $98.41 \mathrm{c}$ & $123.40 c$ & $14.34 \mathrm{c}$ & $17.77 \mathrm{c}$ & $22.22 \mathrm{c}$ & $15.78 c$ & $19.74 c$ & $24.67 c$ \\
\hline LSD 0.05 & 2.80 & 4.40 & 6.30 & 3.10 & 5.10 & 6.50 & 1.00 & 1.10 & 1.30 & 1.05 & 1.10 & 1.45 \\
\hline \multicolumn{13}{|l|}{ Interaction } \\
\hline$A \times B$ & * & * & * & * & * & * & * & * & * & * & * & * \\
\hline
\end{tabular}

Means of each factor designated by the same letter not significantly different at $5 \%$ using least significant difference at $5 \%$ level using (L.S.D.) test *: Significant at 0.05 level of probability. 
Table (6). Interaction between mineral+organic and biofertilization on shoots fresh weight/ plant (g) for moranga plant at three cuts during 2014 and 2015 seasons.

\begin{tabular}{|c|c|c|c|c|c|c|c|}
\hline \multirow{2}{*}{\multicolumn{2}{|c|}{ Treatments }} & \multicolumn{6}{|c|}{ Shoots fresh weight /plant (g) } \\
\hline & & \multicolumn{3}{|c|}{2014 Season } & \multicolumn{3}{|c|}{2015 Season } \\
\hline Org.+mineral & Biofertilization & $1^{\text {st }}$ cut & $2^{\text {nd }}$ cut & $3^{\text {th }}$ cut & $1^{\text {st }}$ cut & $2^{\text {nd }}$ cut & $3^{\text {th }}$ cut \\
\hline \multirow{4}{*}{$100 \%$ org. } & Uninoculation & 72.57 & 90.72 & 11.42 & 80.64 & 100.80 & 126.00 \\
\hline & Phosphorein & 83.29 & 104.11 & 130.16 & 92.54 & 115.80 & 144.60 \\
\hline & Mycorrhizal & 92.10 & 115.13 & 143.81 & 102.33 & 127.92 & 159.90 \\
\hline & Cerealine & 78.10 & 97.63 & 121.98 & 86.78 & 108.48 & 185.60 \\
\hline \multirow{4}{*}{$75 \%$ org. $+25 \%$ mineral } & Uninoculation & 80.64 & 100.30 & 126.00 & 89.60 & 114.66 & 140.00 \\
\hline & Phosphorein & 92.84 & 115.68 & 144.44 & 102.88 & 128.20 & 160.66 \\
\hline & Mycorrhizal & 102.34 & 127.92 & 159.90 & 113.70 & 142.13 & 177.66 \\
\hline & Cerealine & 86.76 & 108.48 & 85.60 & 96.42 & 120.53 & 150.66 \\
\hline \multirow{4}{*}{$50 \%$ org. $+50 \%$ mineral } & Uninoculation & 65.31 & 81.65 & 102.06 & 72.57 & 90.91 & 113.40 \\
\hline & Phosphorein & 74.95 & 93.70 & 117.13 & 83.28 & 104.11 & 130.14 \\
\hline & Mycorrhizal & 92.88 & 103.61 & 129.52 & 92.10 & 115.12 & 143.64 \\
\hline & Cerealine & 70.29 & 87.83 & 109.84 & 78.10 & 97.63 & 122.04 \\
\hline \multirow{4}{*}{$25 \%$ org. $+75 \%$ mineral } & Uninoculation & 58.78 & 73.82 & 91.86 & 65.31 & 81.65 & 102.06 \\
\hline & Phosphorein & 67.46 & 84.32 & 105.42 & 74.95 & 93.65 & 117.12 \\
\hline & Mycorrhizal & 74.59 & 93.25 & 116.57 & 88.88 & 103.60 & 129.51 \\
\hline & Cerealine & 63.26 & 79.08 & 98.86 & 70.29 & 87.86 & 109.83 \\
\hline \multirow{4}{*}{$100 \%$ mineral } & Uninoculation & 52.90 & 66.13 & 82.66 & 58.78 & 73.48 & 91.85 \\
\hline & Phosphorein & 60.73 & 75.89 & 94.87 & 67.46 & 84.99 & 105.41 \\
\hline & Mycorrhizal & 67.14 & 83.92 & 104.91 & 74.59 & 93.25 & 116.56 \\
\hline & Cere & 56.94 & 71.71 & 88.97 & 63.26 & 79.08 & 98.85 \\
\hline \multicolumn{2}{|c|}{ LSD 0.05} & 3.30 & 5.40 & 6.50 & 3.50 & 5.70 & 7.40 \\
\hline
\end{tabular}

Table (7). Interaction between mineral+organic and biofertilization on shoots dry weight/ plant (g) for moranga plants at three cuts during 2014 and 2015 seasons.

\begin{tabular}{|c|c|c|c|c|c|c|c|}
\hline \multirow{2}{*}{\multicolumn{2}{|c|}{ Treatments }} & \multicolumn{6}{|c|}{ Shoots dry weight /plant } \\
\hline & & \multicolumn{3}{|c|}{2014 Season } & \multicolumn{3}{|c|}{2015 Season } \\
\hline Org. + mineral & Biofertilization & $1^{\text {st }}$ cut & $2^{\text {nd }}$ cut & $3^{\text {th }}$ cut & $1^{\text {st }}$ cut & $2^{\text {nd }}$ cut & $3^{\text {th }}$ cut \\
\hline \multirow{4}{*}{$100 \%$ org. } & Uninoculation & 14.52 & 18.14 & 22.68 & 16.13 & 20.16 & 25.20 \\
\hline & Phosphorein & 16.65 & 20.82 & 26.03 & 18.50 & 23.13 & 28.92 \\
\hline & Mycorrhizal & 18.41 & 23.02 & 28.78 & 20.46 & 25.58 & 31.98 \\
\hline & Cerealine & 15.61 & 19.52 & 24.41 & 17.35 & 21.69 & 27.12 \\
\hline \multirow{4}{*}{$75 \%$ org.+25\%mineral } & Uninoculation & 16.12 & 20.16 & 25.20 & 17.91 & 22.40 & 28.00 \\
\hline & Phosphorein & 18.51 & 23.13 & 28.92 & 20.56 & 25.70 & 32.26 \\
\hline & Mycorrhizal & 20.46 & 25.58 & 31.98 & 22.74 & 28.42 & 35.53 \\
\hline & Cerealine & 17.35 & 21.69 & 27.14 & 19.28 & 24.10 & 30.13 \\
\hline \multirow{4}{*}{$50 \%$ org. $+50 \%$ mineral } & Uninoculation & 13.06 & 16.33 & 20.41 & 14.51 & 14.80 & 22.67 \\
\hline & Phosphorein & 14.99 & 18.73 & 23.43 & 16.65 & 20.81 & 26.02 \\
\hline & Mycorrhizal & 16.57 & 20.72 & 25.90 & 18.41 & 23.02 & 28.78 \\
\hline & Cerealine & 14.06 & 17.57 & 21.96 & 15.62 & 19.52 & 24.40 \\
\hline \multirow{4}{*}{$25 \%$ org. $+75 \%$ mineral } & Uninoculation & 11.75 & 14.69 & 18.37 & 13.06 & 16.32 & 20.41 \\
\hline & Phosphorein & 13.49 & 16.86 & 21.41 & 14.99 & 18.74 & 23.45 \\
\hline & Mycorrhizal & 14.92 & 18.65 & 23.31 & 16.52 & 20.72 & 25.40 \\
\hline & Cerealine & 12.65 & 15.82 & 19.79 & 14.05 & 17.57 & 21.90 \\
\hline \multirow{4}{*}{$100 \%$ mineral } & Uninoculation & 10.58 & 13.22 & 16.54 & 11.75 & 14.69 & 18.37 \\
\hline & Phosphorein & 12.14 & 15.18 & 18.97 & 13.49 & 16.86 & 21.08 \\
\hline & Mycorrhizal & 13.42 & 16.78 & 21.03 & 14.91 & 18.64 & 26.70 \\
\hline & Cerealine & 11.38 & 14.23 & 17.79 & 12.62 & 15.81 & 19.76 \\
\hline \multicolumn{2}{|c|}{ LSD (0.05) } & 1.08 & 1.15 & 1.28 & 1.06 & 1.20 & 1.47 \\
\hline
\end{tabular}

Vol. 21(2), 2016 


\section{B) Chemical composition}

Data presented in Tables ( 8 and 9 ) indicated that organic manure plus mineral fertilizers significantly affected nitrogen (\%), phosphorus (\%), potassium $(\%)$, protein (\%), total carbohydrate (\%) and vitamin (C) in both seasons. Application of $75 \%$ organic manure $+25 \%$ mineral; gave rise the highest mean values of all studied chemical composition parameters as compared with application $100 \%$ mineral fertilizer in both seasons.

The increment in chemical composition of moringa leaves using the treatments of organic manure may be owing attributed to increase in the occupancy root zone of plant result of adding organic manure which reflected on $\mathrm{N}, \mathrm{P}$ and $\mathrm{K}$ uptake by plant and confirm the pervious of vegetative growth. Similar results were obtained by Prabhakar and Hebbar (2007), Adebayo et al. (2011) on Moringa oleifera, Makinde (2013) on moringa plant and Attia et al. (2014) on moringa plant.

Concerning the bio-fertilization, treatments in Tables ( 8 and 9) revealed that inoculation moringa plants with bio-fertilization, increased all the studied of chemical composition in both seasons compared to uninoculated moringa (control).

It can, also, be suggested to use combined biofertilizer including phosphorein, A- mycorrhizal and cerealine biofertilizer including all biofertilizer to produce a high quality moringa trees. Several reports on biofertilizer utilization have emphasized that a single inoculation showed higher productivity than uninoculation treatment (control). Shah et al. (2006), Attia et al. (2014) and Mazher et al. (2014).

The interaction between combination organic manure and mineral and bio-fertilization were significant for $\mathrm{N}, \mathrm{P}$ and $\mathrm{K} \%$ in both seasons (Table 10). Application of $75 \%$ organic manure+ $25 \%$ mineral, gave the highest mean values of $\mathrm{N} \%$ with cerealine, $\mathrm{P} \%$ with phosphorein and $\mathrm{K} \%$ with $\mathrm{A}$ - mycorrhizal inoculation as compared with was uninoculation treatment.

The significant differences for the interaction between combination organic manure plus mineral and bio-fertilization in both seasons due to application of $75 \%$ organic manure $+25 \%$ mineral, brought about the greatest protein percentage with treatment of cerealine biofertilizer and total carbohydrate (\%), vitamin (C) with A- mycorrhizal in both seasons (Table 11).

In conclusion, some organs of moringa are good source important minerals and these plants might be explored as a viable supplement and ready source of dietary minerals in animal and human food. There was a significant variation in macro and microelements in moringa leaves. Also, the application of $75 \%$ organic manure $+25 \%$ mineral gave the highest vegetative growth and chemical composition with A- mycorrhizal inoculation. 
Table (8). Nitrogen, phosphorus and potassium percentages as affected by mineral-organic and biofertilization in 2014 and 2015 seasons.

\begin{tabular}{lcccccc}
\hline \multirow{2}{*}{ Treatments } & \multicolumn{3}{c}{ 2014 Season } & \multicolumn{3}{c}{ 2015 Season } \\
\cline { 2 - 7 } & $\mathbf{N} \%$ & $\mathbf{P} \%$ & $\mathbf{K} \%$ & $\mathbf{N} \%$ & $\mathbf{P} \%$ & $\mathbf{K} \%$ \\
\hline A)Mineral + Organic & & & & & & \\
\hline 100\% Organic & $2.91 \mathrm{~b}$ & $0.400 \mathrm{~b}$ & $2.35 \mathrm{~b}$ & $3.23 \mathrm{~b}$ & $0.448 \mathrm{~b}$ & $2.61 \mathrm{~b}$ \\
75\% org. + 25\% mineral & $3.22 \mathrm{a}$ & $0.450 \mathrm{a}$ & $2.61 \mathrm{a}$ & $3.61 \mathrm{a}$ & $0.498 \mathrm{a}$ & $2.90 \mathrm{a}$ \\
$50 \%$ org. + 50\% mineral & $2.62 \mathrm{c}$ & $0.360 \mathrm{c}$ & $2.11 \mathrm{c}$ & $2.91 \mathrm{c}$ & $0.403 \mathrm{c}$ & $2.33 \mathrm{c}$ \\
25\% org. +75\% mineral & $2.35 \mathrm{~d}$ & $0.320 \mathrm{~d}$ & $1.91 \mathrm{~d}$ & $2.61 \mathrm{~d}$ & $0.355 \mathrm{~d}$ & $2.12 \mathrm{~d}$ \\
100\% mineral & $2.12 \mathrm{e}$ & $0.290 \mathrm{e}$ & $1.96 \mathrm{~d}$ & $2.35 \mathrm{e}$ & $0.318 \mathrm{e}$ & $2.17 \mathrm{~d}$ \\
\hline LSD 0.05 & $\mathbf{0 . 1 0}$ & $\mathbf{0 . 0 1 2}$ & $\mathbf{0 . 1 1}$ & $\mathbf{0 . 1 1}$ & $\mathbf{0 . 0 4 0}$ & $\mathbf{0 . 1 9}$ \\
\hline B) Bio-fertilization & & & & & & \\
\hline Uninoculation & $2.19 \mathrm{c}$ & $0.250 \mathrm{~d}$ & $2.04 \mathrm{~d}$ & $2.43 \mathrm{c}$ & $0.280 \mathrm{~d}$ & $2.16 \mathrm{c}$ \\
Phosphorein & $2.57 \mathrm{~b}$ & $0.472 \mathrm{a}$ & $2.15 \mathrm{c}$ & $2.85 \mathrm{~b}$ & $0.520 \mathrm{a}$ & $2.38 \mathrm{~b}$ \\
Mycorrhizal & $2.67 \mathrm{~b}$ & $0.410 \mathrm{~b}$ & $2.40 \mathrm{a}$ & $2.92 \mathrm{~b}$ & $0.452 \mathrm{~b}$ & $2.66 \mathrm{a}$ \\
Cerealine & $3.16 \mathrm{a}$ & $0.326 \mathrm{c}$ & $2.25 \mathrm{~b}$ & $3.51 \mathrm{a}$ & $0.364 \mathrm{c}$ & $2.48 \mathrm{~b}$ \\
\hline LSD 0.05 & $\mathbf{0 . 1 1}$ & $\mathbf{0 . 0 1 5}$ & 0.08 & $\mathbf{0 . 1 2}$ & $\mathbf{0 . 0 5 0}$ & $\mathbf{0 . 1 7}$ \\
\hline Interaction & & & & & & \\
\hline AxB & $*$ & $*$ & $*$ & $*$ & $*$ & $*$ \\
\hline
\end{tabular}

Means of each factor designated by the same letter not significantly different at $5 \%$ using least significant difference at $5 \%$ level using (L.S.D.) test

*: Significant at 0.05 level of probability.

Table (9). Protein (\%), vitamin (C) and total carbohydrate (\%) as affected by mineral-organic and biofertilization in 2014 and 2015 seasons.

\begin{tabular}{|c|c|c|c|c|c|c|}
\hline \multirow[b]{2}{*}{ Treatments } & \multicolumn{3}{|c|}{2014 Season } & \multicolumn{3}{|c|}{2015 Season } \\
\hline & $\begin{array}{c}\text { Protein } \\
\%\end{array}$ & $\begin{array}{l}\text { Vitamin } \\
\text { (C) } \\
\text { mg/100 } \\
\text { ml juice }\end{array}$ & $\begin{array}{c}\text { Total } \\
\text { carbohydrate } \\
\%\end{array}$ & $\begin{array}{c}\text { Protein } \\
\%\end{array}$ & $\begin{array}{c}\text { Vitamin } \\
\text { (C) } \\
\text { mg/100 } \mathrm{ml} \\
\text { juice } \\
\end{array}$ & $\begin{array}{c}\text { Total } \\
\text { carbohydrate } \\
\%\end{array}$ \\
\hline \multicolumn{7}{|l|}{ A) Mineral + Organic } \\
\hline $100 \%$ Organic & $18.60 \mathrm{~b}$ & $0.526 b$ & $19.79 b$ & $20.20 b$ & $0.584 b$ & $22.06 \mathrm{~h}$ \\
\hline $75 \%$ org. $+25 \%$ & $20.22 a$ & $0.584 a$ & $22.06 a$ & $22.47 a$ & $0.648 a$ & $24.51 a$ \\
\hline mineral $50 \%$ org. $+50 \%$ mineral & $16.36 c$ & $0.467 c$ & $17.87 \mathrm{c}$ & $18.18 c$ & $0.519 c$ & $19.85 c$ \\
\hline $25 \%$ org. $75 \%$ mineral & $14.71 d$ & $0.420 d$ & $16.10 d$ & $16.34 d$ & $0.467 d$ & $17.87 d$ \\
\hline $100 \%$ mineral & $13.25 \mathrm{e}$ & $0.409 d$ & $14.47 \mathrm{e}$ & $14.70 \mathrm{e}$ & $0.454 d$ & $16.08 \mathrm{e}$ \\
\hline LSD 0.05 & 1.10 & 0.35 & 1.30 & 1.20 & 0.052 & 1.45 \\
\hline \multicolumn{7}{|l|}{ B) Bio-fertilization } \\
\hline Uninoculation & $14.02 \mathrm{c}$ & $0.449 d$ & $14.27 d$ & $15.20 \mathrm{c}$ & $0.497 c$ & $15.85 d$ \\
\hline Phosphorein & $16.05 b$ & $0.497 b$ & $19.02 b$ & $17.83 b$ & $0.552 a$ & $21.14 b$ \\
\hline Mycorrhizal & $16.67 b$ & $0.514 a$ & $22.03 a$ & $18.51 b$ & $0.570 a$ & $24.54 a$ \\
\hline Cerealine & $19.70 \mathrm{a}$ & $0.466 \mathrm{c}$ & $16.90 \mathrm{c}$ & $21.95 a$ & $0.518 b$ & $18.78 \mathrm{c}$ \\
\hline LSD (0.05) & 1.20 & 0.015 & 1.50 & 1.30 & 0.040 & 1.90 \\
\hline \multicolumn{7}{|l|}{ Interaction } \\
\hline AxB & * & * & * & * & * & * \\
\hline
\end{tabular}

Means of each factor designated by the same letter not significantly different at $5 \%$ using least significant difference at $5 \%$ level using (L.S.D.) test

*: Significant at 0.05 level of probability. 
Table (10). Interaction between mineral+organic and biofertilization on macronutrients (N, P and K \%) for moranga plants in 2014 and 2015 seasons.

\begin{tabular}{|c|c|c|c|c|c|c|c|}
\hline \multicolumn{2}{|l|}{ Treatmen } & \multicolumn{2}{|c|}{ N\% } & \multicolumn{2}{|c|}{$\mathbf{P} \%$} & \multicolumn{2}{|c|}{$\mathrm{K} \%$} \\
\hline Org. + mineral & Biofertilization & 2014 & 2015 & 2014 & 2015 & 2014 & 2015 \\
\hline \multirow{4}{*}{$100 \%$ Org. } & Uninoculation & 2.41 & 2.67 & 0.28 & 0.31 & 2.13 & 2.36 \\
\hline & Phosphorein & 2.83 & 3.13 & 0.52 & 0.58 & 2.36 & 2.62 \\
\hline & Mycorrhizal & 2.93 & 3.25 & 0.45 & 0.50 & 2.64 & 2.93 \\
\hline & Cerealine & 3.48 & 3.66 & 0.36 & 0.40 & 2.27 & 2.51 \\
\hline \multirow{4}{*}{$75 \%$ org. $+25 \%$ mineral } & Uninoculation & 2.68 & 2.97 & 0.30 & 0.34 & 2.87 & 2.63 \\
\hline & Phosphorein & 3.14 & 3.49 & 0.59 & 0.65 & 2.62 & 2.91 \\
\hline & Mycorrhizal & 3.26 & 3.62 & 0.50 & 0.55 & 2.93 & 3.26 \\
\hline & Cerealine & 5.86 & 4.29 & 0.40 & 0.45 & 2.53 & 2.80 \\
\hline \multirow{4}{*}{$50 \%$ org. $+50 \%$ mineral } & Uninoculation & 2.16 & 2.40 & 0.25 & 0.28 & 1.92 & 2.13 \\
\hline & Phosphorein & 2.54 & 2.82 & 0.47 & 0.52 & 2.12 & 2.33 \\
\hline & Mycorrhizal & 2.64 & 2.93 & 0.40 & 0.45 & 2.37 & 2.63 \\
\hline & Cerealine & 3.13 & 3.47 & 0.32 & 0.36 & 2.04 & 2.21 \\
\hline \multirow{4}{*}{$25 \%$ org. $+75 \%$ mineral } & Uninoculation & 1.94 & 2.16 & 0.22 & 0.25 & 1.73 & 1.92 \\
\hline & Phosphorein & 2.28 & 2.54 & 0.41 & 0.45 & 1.91 & 2.12 \\
\hline & Mycorrhizal & 2.37 & 2.63 & 0.57 & 0.40 & 2.13 & 2.37 \\
\hline & Cerealine & 2.81 & 3.12 & 0.29 & 0.32 & 1.85 & 2.05 \\
\hline \multirow{4}{*}{$100 \%$ mineral } & Uninoculation & 1.75 & 1.94 & 0.20 & 0.22 & 1.57 & 1.74 \\
\hline & Phosphorein & 2.06 & 2.28 & 0.37 & 0.40 & 1.75 & 1.94 \\
\hline & Mycorrhizal & 2.13 & 2.37 & 0.33 & 0.36 & 1.92 & 2.13 \\
\hline & Cerealine & 2.53 & 2.81 & 0.26 & 0.29 & 2.58 & 2.86 \\
\hline \multicolumn{2}{|c|}{ LSD (0.05) } & 0.13 & 0.15 & 0.017 & 0.06 & 0.14 & 0.20 \\
\hline
\end{tabular}

Table (11). Interaction between mineral+organic and biofertilization on Protein \%, Vitamin (C) and Total carbohydrate \% for moranga plants in 2014 and 2015 seasons.

\begin{tabular}{|c|c|c|c|c|c|c|c|}
\hline \multicolumn{2}{|c|}{ Treatments } & \multicolumn{2}{|c|}{ Protein (\%) } & \multicolumn{2}{|c|}{$\begin{array}{c}\text { Vitamin (C) } \\
\text { ( } \mathrm{mg} / 100 \mathrm{ml} \text { juice ) }\end{array}$} & \multicolumn{2}{|c|}{$\begin{array}{c}\text { Total } \\
\text { carbohydrate (\%) }\end{array}$} \\
\hline Org. + mineral & Biofertilization & 2014 & 2015 & 2014 & 2015 & 2014 & 2015 \\
\hline \multirow{4}{*}{$100 \%$ Org. } & Uninoculation & 16.72 & 16.72 & 0.488 & 0.543 & 15.68 & 17.42 \\
\hline & Phosphorein & 17.64 & 19.60 & 0.543 & 0.604 & 20.89 & 23.22 \\
\hline & Mycorrhizal & 18.32 & 20.35 & 0.562 & 0.624 & 24.00 & 26.97 \\
\hline & Cerealine & 21.73 & 24.14 & 0.509 & 0.566 & 18.57 & 20.64 \\
\hline \multirow{4}{*}{$75 \%$ org. $+25 \%$ mineral } & Uninoculation & 16.74 & 18.60 & 0.542 & 0.603 & 17.42 & 19.36 \\
\hline & Phosphorein & 19.63 & 21.81 & 0.604 & 0.671 & 23.22 & 25.80 \\
\hline & Mycorrhizal & 20.36 & 22.62 & 0.624 & 0.688 & 26.47 & 29.96 \\
\hline & Cerealine & 24.16 & 26.85 & 0.566 & 0.629 & 20.64 & 22.93 \\
\hline \multirow{4}{*}{$50 \%$ org. $+50 \%$ mineral } & Uninoculation & 13.53 & 15.03 & 0.434 & 0.483 & 14.11 & 15.68 \\
\hline & Phosphorein & 15.88 & 17.64 & 0.482 & 0.536 & 18.80 & 20.89 \\
\hline & Mycorrhizal & 16.49 & 18.31 & 0.494 & 0.554 & 21.84 & 24.27 \\
\hline & Cerealine & 19.55 & 21.77 & 0.452 & 0.503 & 16.72 & 18.57 \\
\hline \multirow{4}{*}{$25 \%$ org. $+75 \%$ mineral } & Uninoculation & 12.16 & 13.61 & 0.396 & 0.434 & 12.70 & 14.11 \\
\hline & Phosphorein & 14.28 & 15.87 & 0.434 & 0.482 & 16.98 & 18.80 \\
\hline & Mycorrhizal & 14.83 & 16.47 & 0.450 & 0.499 & 19.66 & 21.84 \\
\hline & Cerealine & 17.57 & 19.52 & 0.407 & 0.452 & 15.04 & 16.71 \\
\hline \multirow{4}{*}{$100 \%$ mineral } & Uninoculation & 10.93 & 12.14 & 0.380 & 0.422 & 11.43 & 12.09 \\
\hline & Phosphorein & 12.84 & 14.27 & 0.422 & 0.469 & 15.23 & 16.91 \\
\hline & Mycorrhizal & 13.33 & 14.81 & 0.436 & 0.480 & 17.69 & 19.66 \\
\hline & Cerealine & 15.80 & 17.56 & 0.396 & 0.440 & 13.54 & 15.04 \\
\hline \multicolumn{2}{|c|}{ LSD (0.05) } & 1.22 & 1.33 & 0.040 & 0.055 & 1.35 & 1.96 \\
\hline
\end{tabular}

Vol. 21(2), 2016 


\section{REFERENCES}

Abou El-Fadi, M., S. G. Rizk, A. F. Abdel Ghani, M. K. El-Mofty, M. E. A. Khadr, S. M. Shehata nd F. A. Farg (1968). Utilization of water hyacinth as an organic manure with special reference to water-brone helminthes. J. Microbial A.R.F., 3(1):27-34.

Adebayo, A.G., H.A. Akintoye, O.O. Olufolaji, M.T. Aina, M.T. Olatunji and A.O. Shokalu (2011). Assessment of organic amendments on vegetative development and nutrient uptake of Moringa oleifera Lam in the nursery. Asian J. Plant Sci., 10(1):74-79.

Adeoye, D. A. and A. A. Aghools (2005). Critical level of soil plant available P, $\mathrm{K}, \mathrm{Zn}, \mathrm{Mg}, \mathrm{Cu}$ and $\mathrm{Mn}$ on maize leaf content of sedimentary soil of South West Nigeria. Fert. Res., 6: 66-71.

Anjorin, T. S., P. Ikokoh nd S. Okolo (2010). Mineral composition of Moringa oleifera leaves, pods and seed from two regions in Abuja Nigeria. Int. J. Agric. \& Biol., 3:431-434.

AOAC (1990). Official method of analysis, fifteenth ed. association of official analytical chemists, Virginia, USA.

AOAC (1984). Official Method of Analysis of the Association of Official Analytical Chemists, 14thed,Published by the Association of Official Analytical Chemists, PO Box,540, Benjamin Franklin Station, Washington, dc.20044.

Attia, M. F., M.F.M. Shahin, M.A. Merwad, E. S. El-Hady and L. F. Haggag (2014). Effect of mineral, organic and bio-fertilization on productivity of moringa plant under saline conditions in North Sinai. Mid. East J. Appl. Sci., 4(4): 825-832.

Chapman, H. D. and P.F. Pratt (1978). Method of Analysis for Soil and Water. 2nd Ed., Chapter, 17:150-161. Uni. Calif. Div. Agric. Sci. USA.

Dania, S. O.; P. Akpansubi and O. O. Eghagara (2014). Comparative effects of different fertilizer sources on the growth and nutrient content of moringa (Moringa oleifera) seedling in a greenhouse trial. Adv. Agric., 1-6

Gomez, K. A. and A. A. Gomez (1984). Statistical procedure for agricultural research, jhon willey and sons. Inc. New York.

Ismali, A. G., E. M. Desouky, Y. Gomal, M. Gall, A. A. Arafa and Abou-Seer (2009). Effect of biofertilizers and organic phosphorus mendments on growth and essential oil of marjoram. Egypt Acad. J. Biol. Sci., 1(1):2930.

Jackson, M.L. (1973). Soil Chemical Analysis. Prentice Hall of India. Private Limited, New Delhi, India, pp. 183-192.

Lowther, J. R. (1980). Use of a single sulphuric-hydrogen peroxide digest for the analysis of Pinus radiate needles. Commun. Soil Sci. Plant Anal. 11: 175-188.S

Makinde, A. I. (2013). Effects of inorganic fertilizer on the growth and nutrient composition of moringa (Moringa oleifera). J. Emer. Trend Eng. \& Appl. Sci., 4(2): 341-343.

Manorama, S., S. Paulsamy and D. Suresh (2007). Evaluation of seedlings of six tree species in lime mined soil by using some amendments. Range Manag. \& Agrofor., 28(1): 21-24. 
Mazher, A. A. M., N. G. Abdel-Aziz, R. S. El-Dabh, M. A. El-Khateeb and A. A. Abd El-Badaie (2014). Effect of Bio Fertilization on Growth and Constituents of Moringa oleifera Lam. Plants. Middle East J. Agric. Res., 3(4): 793-798.

Morton, J. F. (1991). The horseradish tree, Moringa pterygosperma (Moringaceae) a boon to arid lands. Econ. Bot., 45: 318-333.

Prabhakar, M. and S.S.Hebbar (2007). Studies on organic production technology of annual drumstick in a semiarid agro-ecosystem. ISHS Acta Hort., 752(1): 117, Hyderabad, India.

Rajendrn, K., V. Sugavanam and P. Devaraj (2000). Influence of biofertilizers on the biomss production of Cassuasin equisetifolia farm forestry. Bngladish J. Forest Sci., 29(1):26-36.

Shah, S. K.; R. P. Shah; H. L. Xu and U. K. Aryal (2006). Biofertilizers: an alternative source of nutrients for sustainable production of tree crops. J. Sustainable Agric., 29(2): 85-95.

Yemm, E.W. and A. J. Willis (1954). The estimation of carbohydrate in extracts by anthrone. Biochem. J., 57: $508-514$. 


\section{الملخص العربي}

\section{تأثير التسميد المعدني والعضوي والحيوي علي نمو وإنتاج نباتات المورينجا} أ.د. فتحي إبراهيم رضوان أ.د. علي إبراهيم علي عيبدو د. العيد حسن حسين شعبان نزار عبد الخالق عبد الجبار

قسم الإنتاج النباتي - كلية الزراعة (سابا بانثا) - جامعة الإسكندرية

أجريت تجربتان حقليتان في المزرعة البحثية بكلية الزراعة (سابا باشا) جامعة الأسكندرية-منطقة أبيس - حمهورية

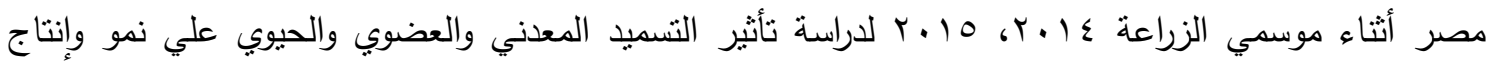
نباتات المورينجا.

صمدت التجربة بتصميم القطع المنشقة مرة واحدة مع ثلاث مكرارت وكانت القطع الريئسية عبارة عن

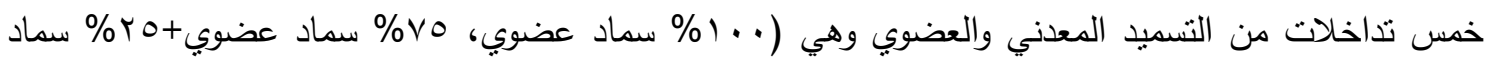

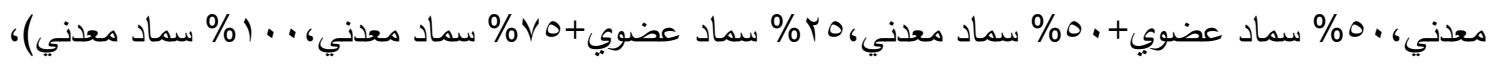
بينما أربع معاملات تسميد حيوي (بدون تلقيح، فوسفورين، ميكوريزا، سيريالين) كانت موزعة في القطع المنشقة الأولي.

وكانت من أهم النتائج المتحصل عليها مايلي:

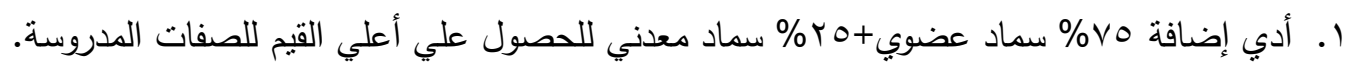

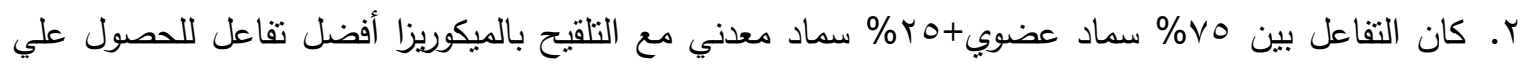

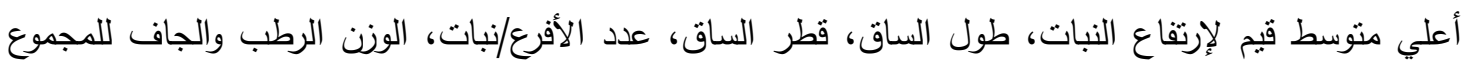

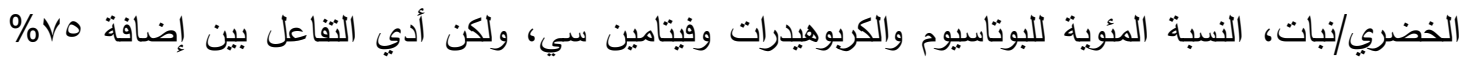

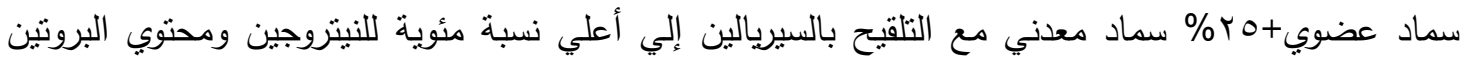

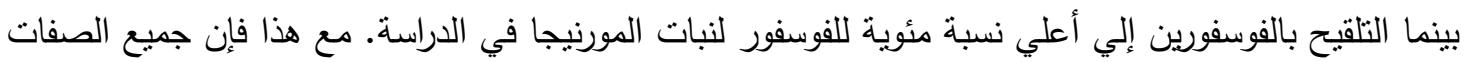

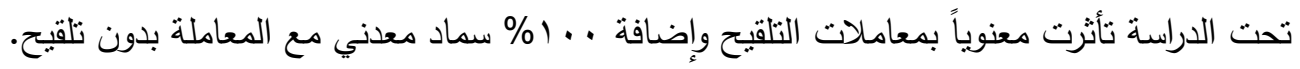


